\title{
Programação Linear por Partes: Revisão Teórica e Aplicações
}

\section{Fernando Augusto Silva Marins}

Departamento de Produção, Faculdade de Engenharia do Campus de Guaratinguetá UNESP, CEP 12500-000, Guaratinguetá, SP, Brasil

\section{Clóvis Perin Filho}

Departamento de Matemática Aplicada, Instituto de Matemática e Estatística

UNICAMP, CEP 13081-970, Campinas, SP, Brasil.

Palavras-Chaves: Programação linear por partes; programação em redes lineares por partes; programação linear e programação não-linear.

Key Words: Piecewise-Linear Programming, Network Piecewise-Linear Programming

\section{RESUMO}

Procura-se resgatar a importância de uma subarea da Programação Malemática conlyecida como Programação Linear Por Partes - PLP. De fato a PLP tem inúmeras aplicações tanto na área teórica como em situações reais. Este trabalho apresenta os resultados de uma pesquisa bibliogrática, etetuada nas principais revistas técnicas e livros disponiveis relacionados com Pesquisa Operacional, que visou situar o estado da' arte da Programação Linear por Partes, bem cono a abrangència de sua aplicabilidade. Particularmente, no contexto da PLP, este texto destaca a Programação em Redes Lincares por Partes devido a sua relevância em muitas situaçōes práticas.

\section{ABSTRACT}

The importance of a subarea of Mathematical Programming known as Piecewise-Linear Programming - PLP is emphasized. In fact PLP has many both theoretical and real life applicalions. This paper presents the results of an extensive bibliographical research, including the most relevant Operations Research journals and books available, which had as goal to find out Piecewise-Linear Programming state-of-art, as well as its range of applicability. Particularly, inside PL $P$ context. this paper details Network Piecewise-Linear Programming due to its relevance to several real life situations. 


\section{Introdução}

O objetivo principal deste trabalho é o de divulgar e motivar as pesquisas em Programação Linear por Partes - PLP, cuja importância fica evidente ao se constatar suas inúmeras aplicações. Inicialmente, procura-se situar a PLP em geral, explicitando sua importância tanto do ponto de vista teórico como prático através de uma revisão comentada de alguns dos principais traballıos desenvolvidos na área. Particularmente, a seguir, é destacada a sub-área da PLP denominada Programação em Redes Lineares por Partes - PRLP que, apesar de sua aplicabilidade, não tem sido devidamente explorada e oferece opções de desenvolvimento de pesquisas de relevância.

\section{Origens e Revisão da Literatura em Progra- mação Linear Por Partes}

Uma importante área da Programação Matemática é a Programação Linear Por Partes - PLP. Os modelos de PLP, que fornecem uma formulação natural para muitos problemas de otimização, tratam da minimização de uma função objetivo separável e linear por partes, respeitando un conjunto de restrições representadas por um sistema de equações lineares. Portanto, nestes modelos de PLP, a função objetivo é uma soma de funções lineares por partes, cada uma das quais expressa com respeito a apenas uma das variáveis do problema.
Existem inúmeras aplicações conhecidas para a PLP das quais passa-se a comentar brevemente as que se seguem:

(a) Aplicação da PLP na determinação de solução viável inicial em Programação Linear - PL: isto é feito através da resolução de um programa linear por partes, cuja solução ótima é uma solução viável para o programa linear original. Esta é a aplicação mais comum e conhecida da PLP, ver por exemplo, Orchard-Hays ${ }^{64}$, tradicionalmente usada em códigos comerciais com implementações do método simplex apropriados para problemas de grande porte;

(b) Aplicação da PLP como um método de penalidades: é um procedimento ben conhecido, que acrescenta uma penalidade linear por partes à função objetivo do programa linear original. Isto é feito de tal forma que toda solução viável e ótima do programa linear original é também uma solução ótima para o problema com a penalidade. Comn ${ }^{13} \mathrm{e}$ Bartels ${ }^{7}$ propuseram algoritmos especiais tipo simplex para este tipo de formulação de programas;

(c) Aplicação da PLP em atividades reversiveis: certas siluações envolvem atividades (ou variáveis) que fazem sentido tanto quando colocadas em niveis positivos como negativos, como é o caso de sistemas de estoques, com possibilidade de haver pedidos pendentes ("backorder"). Os custos associados a este tipo de variáveis podem ser representados 


\section{PRODUÇÃO}

por funções lineares por partes, apresentando termos lineares com inclinações (ou coeficientes de custo) negativas onde as atividades forem negativas, $\mathrm{e}$ inclinações positivas onde as atividades forem positivas;

(d) Aplicação da PLP em problemas de estimação L1: em determinados problemas de estimação (ajuste de curvas) busca-se determinar, em termos da norma $\mathrm{Ll}$, a solıção aproximada mais acurada para um sistema de equações lineares $\mathrm{Ax}=\mathrm{b}$. A partir da introdução de variáveis de folga estes problemas podem ser expressos como um caso particular da PLP, onde, no modelo linear por partes, as variáveis originais $x$ terão coeficientes de custo nulos na função objetivo linear por partes, e as variáveis de folga terão coeficientes de custo - I (ou +1 ) quando elas forem negativas (ou positivas). Várias generalizações deste problema de estimação $L 1$ são também passiveis de ser representadas por programas lineares por partes, confonie comentado por Fourer ${ }^{25}$, e existem inúmeros algoritmos especializados tipo simplex desenvolvidos para estas situações, tais como os descritos por Davies ${ }^{18}$. Barrodale \& Roberts ${ }^{6}$, e Spyropoulos et al. ${ }^{81}$ que foram os pioneiros neste enfoque;

(e) Aplicação da PLP na Programação por Metas ("Goal Programming"): para o caso da minimização da soma ponderada dos desvios, que podem ser positivos ou negativos, do produto Ax com respeito a sua igualdade com o vetor de constantes b, sujeito a restrições lineares adicionais sobre $x$, este modelo de programação por metas pode ser estabelecido como sendo um modelo linear por partes. Neste caso, todas as variáveis do modelo linear por partes terão dois intervalos de variação ($\infty, 0]$ e $[0,+\infty)$ para seus valores admissiveis. As variáveis originais $x$ terão coeficientes de custo - $\infty$ e coeficientes de custo 0 , respectivamente no primeiro e segundo intervalos. Já as variáveis de folga (desvios) terão coeficientes de custo dados, no intervalo $(-\infty, 0]$ pelo peso usado na ponderação dos desvios positivos, e no intervalo $(0,+\infty)$ pelo peso adolado para os desvios negativos:

(f) Aplicação da PLP em Programação Linear com lariáveis e Restrições Canalizadas: esta é a situação de problemas lineares onde existem limitantes inferiores e superiores tanto para as variáveis como também para as restrições envolvidas. Ocorre que, conforme comentado por Fourer ${ }^{41}$, pela teoria da dualidade da PLP existe um programa linear por partes que é o dual deste problema com variáveis e restrições canalizadas, o que permite enfoques alternativos para o caso. Existem trabalhos que exploram estas caracleristicas particulares destes modelos primal e dual e apresentam algoritmos especializados, como os de Robers \& Bell-Israel ${ }^{70}$ e o de Gunn ${ }^{37}$;

(g) Aplicação da PLP à Programação Não-Linear - PNL : um procedimento simplificador, muito aplicado, no estudo de problemas em Programação Não-Linear 
Separável, tem sido o de substituir os termos não lineares por aproximações lineares por partes, como proposto por Charnes \& Lemke', Charnes \& Cooper ${ }^{10}$, Dantzig $^{16}$, e Dantzig et al. ${ }^{17}$. Em certos casos, este método pode não produzir um problema convexo separável linear por partes equivalente, pois a função objetivo resultante pode não ser convexa, ou, ainda, podem surgir restrições lineares por partes. Contudo, algumas situações relevantes neste contexto podem ser tratadas pela PLP, como descrito por Fourer ${ }^{25}$.

Os interessados na obtenção de maiores detalhes sobre estas, e outras, aplicações da PLP podem consultar as referências Marins ${ }^{57}$ e Fourer ${ }^{25}$.

Com respeito à literatura consultada, deve-se destacar uma seqüência de quatro excelentes artigos, Fourer ${ }^{25,26,27}$ e Fourer $\&$ Marsten $^{28}$, que resgataram a relevância da PLP no cenário científico. O primeiro artigo introduz, de forma rigorosa, os conceitos de funções convexas separáveis e lineares por partes e de dualidade, estabelecendo a seguir as propriedades de um programa linear por partes, a definição de solução básica, e finalmente descrevendo um algoritmo especializado para a PLP que toma vantagem das características deste tipo de modelo para se tornar mais vantajoso, do ponto de vista computacional, do que outros enfoques alternativos.

Nos segundo e terceiro traballıos são apresentados, e discutidos, resultados teóricos sobre convergência ("finiteness") e validade ("correcteness") do algoritmo, questões sobre viabilidade e o seu comportamento frente a ocorrência de degenerescência.Também é feita uma análise teórica das vantagens computacionais de se aplicar o algoritmo desenvolvido para a PLP. Já no último artigo, os autores descrevem os resultados de um interessante estudo computacional realizado envolvendo o algoritmo de Fourer e um algoritmo tipo simplex bem conhecido. Este estudo comprova as vantagens do uso de um algoritmo especializado para PLP.

Adolando os critérios considerados por Fourer, nos trabalhos acima citados, há três enfoques para a PLP quie vêm à classificar as pesquisas desenvolvidas nesta área em três categorias.

Primeiramente, a PLP como caso especial da Programação Linear. De fato, este tem sido o tratamento tradicional dado a modelos de PLP, que são então considerados como apenas mais uma aplicação da PL. Assim o procedimento usualmente aplicado é o de converter o modelo de PLP em um modelo linear equivalente, de maior porte, através de transformações conhecidas, como as propostas por Charnes \& Lemke", Dantzig $^{16}$, Dantzig et al. ${ }^{17}$, e $\mathrm{Ho}^{38}$. Este modelo linear equivalente é então resolvido por um algoritmo tipo simplex, ou ainda, por meio de algum algoritmo simplex adaptado para explorar certos aspectos particulares do modelo linear equivalente obtido. Uma descrição sucinta dos algoritmos publicados por Snyder ${ }^{77}$. ${ }^{78}$. e Premoli ${ }^{67}$. que abordam este último tipo 
de enfoque, pode ser encontrada em Marins $^{57}$.

Como apresentado por $\mathrm{Ho}^{38}$, há pelo menos quatro métodos equivalentes para linearizar problemas convexos separáveis lineares por partes. Cada uma destas transformações leva a diferentes formulações em PL, evidentemente todas com soluções ótimas equivalentes. Os procedimentos mais conhecidos são o método e o-método que trabalham com as variáveis do problema, além do -método e do -método que utilizam diferentes representações para a função objetivo.

É importante observar que os modelos lineares equivalentes obtidos pelos procedimentos acima possuem um número muito maior de variáveis e restrições que o modelo linear por partes original. De fato, estes métodos definem ao menos uma variável e/ou restrição adicional para cada termo linear de todas as parcelas que forman a função objetivo convexa, separável e linear por partes original.

Um segundo enfoque dos trabalhos na área consiste em considerar a PLP como caso especial da Programação NãoLinear - PNL. Vários pesquisadores têm estudado importantes problemas da PNL para os quais a PLP é extremamente útil. Particularmente na área de Programação Separável, como já comentado, podem ser encontrados relevantes resultados teóricos e práticos obtidos usando a linearização por partes. Alguns destes resultados estão descritos nos trabalhos de Allfeld et al. ${ }^{1}$, Geoffrion ${ }^{31}$, Güder \&
Morris $^{36}$, Hochbaum \& Shanthikumar ${ }^{39}$, Kamesam \& Meyer ${ }^{40}$, Kao e Meyer ${ }^{41}$. Meyer $^{59}$, Meyer ${ }^{60}$. Rockafellar ${ }^{72}$, e de Thakur ${ }^{84,85}$, comentados a seguir.

Geoffrion estabeleceu um critćrio natural para selecionar a "melhor" aproximação para uma função objetivo não-linear, e Thakurs apresenta um estudo sobre o erro de truncamento que ocorre no valor ótimo da função objelivo devido ao uso de aproximações lineares por partes. Particularmente, Güder \& Morris ${ }^{36}$ apresentam um método para obter a aproximação linear por partes ótima de uma função objetivo convexa, separável e quadrática, e recomenda o uso de algoritmos especializados para resolver o modelo de PLP resultante.

O artigo de Ahlfeld et al. ' descreve uma especialização do algoritmo truncado de Newton para problemas não-lineares em redes generalizadas, e o uso de uma linearização por partes na obtenção de una solução viável inicial. Os demais trabalhos sugerem ou a idéia de converter o programa linear por partes em um programa linear equivalente. e então aplicar um algoritmo de otimização linear, ou propõem o uso de algoritmos não-lineares especializados para a solução direla do modelo linear por partes.

Pode-se, ainda, citar, como relacionados com a PLP na área de PNL, importantes trabalhos em Otimização Monotrópica

("Monotropic Optimisation"), como o de Rockafellar ${ }^{71}, o$ de Snyder ${ }^{77}$ em métodos do gradiente 
reduzido para Otimização sob Restrições, os de Bartels ${ }^{7}$ e de Comn ${ }^{13}$, na solução de problemas de estimação $L_{1}$, e os de Markowitz \& Perold ${ }^{58}$ e Perold ${ }^{66}$ para problemas de otimização de "portfolios" com critério quadrático.

Uma terceira categoria de trabalhos envolvendo modelos de PLP, trata a PLP como generalização da PL. Os artigos de Fourer $^{25.26 .27}$, Fourer \& Marsten ${ }^{28}$, e o capiltulo 7 do livro de Golstein \& Youdine ${ }^{35}$ descrevem em detalhes vários aspectos teóricos ligados a esta visão da PLP.

Deve-se observar que alguns artigos publicados há algum tempo, como os de Golstein $^{34}$ e Orden \& Nalbandian ${ }^{65}$, já propunham algoritmos especializados para modelos de PLP. Além destes autores, vários pesquisadores, tais como $\mathrm{Beale}^{8}$, Dantzig's, Ferguson \& Dantzig ${ }^{22}$, Williams $^{87}$ eII Programação Linear sob Incerteza, Charret" e Rosvany' ${ }^{74.75} \mathrm{em}$ Projeto Estrutural, publicaram descrições de muitas e variadas situações onde modelos de PLP desempenlam importante papel.

Uma ampla pesquisa bibliográfica acerca deste enfoque para a PLP pode ser encontrada em Marins ${ }^{57}$, onde se incluem os temas de várias teses de mestrado e doutoramento (Fernandes ${ }^{23}$, Ferreira ${ }^{24}$. Garcia $^{30}$, Ribeiro ${ }^{69}$, e Souza ${ }^{80}$ ) desenvolvidas na Faculdade de Engenharia Elétrica da Universidade Estadual de Campinas, no periodo de 1977 a 1979, que abordam a área em questão. A idéia principal deste último tratamento da
PLP é o desenvolvimento de teoria e algoritmos próprios para a PLP, através da extensão natural dos conceitos e definições válidas para a PL.

Com respeito a PLP em geral, deve-se, ainda, referenciar o artigo de De Wolf et al. ${ }^{20}$ no qual os autores considerando problemas separáveis, onde funções nãolineares também podem aparecer nas restrições, propõem o tratamento destes problemas pela PLP. Eles citam como exemplos de aplicação problemas de transmissão de gás com perdas na tubulação, e outras situações similares, como problemas de distribuição de água com perdas, e problemas de simulação de transmissão de eletricidade usando uma aproximação das equações de fluxo de carga ell corrente continua.

\section{A Programação em Redes Lineares porPartes}

O uso de modelos em redes tem crescido substancialmente nos últimos anos, dado o seu reconhecimento como uma importante ferramenta para problemas associados com a Teoria da Decisão. Como comentado por Glover et al. ${ }^{32}$, este fenômeno é devido a três propriedades inerentes aos modelos de redes:

(a) Fácil Vizualização de fato as relações relevantes existentes no problenta podell ser convenientemente representadas por meio de diagramas (grafos) que permitem uma identificação rápida por parte do usuário do modelo;

(b) Flexibilidade de Modelo. como pode ser constatado na abundante 
literatura existente sobre modelos em redes (ver, por exemplo, Glover et al ${ }^{42,43}$ e Ahuja et al. ${ }^{3}$, Mulvey ${ }^{62}$, e Veldhorst ${ }^{86}$ ), estes modelos sĩo extremamente úteis e cobrem uma enorme diversidade de aplicações em variadas áreas;

(c) Facilidade de Solução, devido às suas características particulares, a proposição de novas estruturas de armazenamento de dados extremamente eficientes, e ao surgimento de enfoques alternativos para o desenvolvimento de algoritmos, atualmente existem disponíveis inúmeros métodos de solução especializados para modelos em redes, inclusive para as de grande porte, possibilitando a solução direta de muitos problemas que, até recentemente, não podiam ser resolvidos em tempo razoável.

Esta importância de modelos em redes tem atraído a atenção de pesquisadores que vêm desenvolvendo novos mélodos, para a abordagem das muitas classes de modelos em redes conhecidas. Unt texto bem atualizado, e interessante, que oferece uma descrição completa de algoritmos e classes de problemas em redes, é o livro publicado por Aluuja et al. ${ }^{3}$.

Na interface da PLP e da Programação em Redes está a Programação em Redes Lineares por Partes - PRLP. Os modelos da PRLP têm como meta a minimização de uma função objetivo convexa, separável e linear por partes, sujeita a um sistema de equações lineares que expressam a conservação de fluxo sobre uma matriz de incidência de uma rede conectada, que representa as relações existentes entre as variáveis do problema. Ao contrário do que ocorre para modelos de redes lineares, cujo tratamento teórico e estado da arte dos algoritmos disponiveis têm sido extensamente divulgados, muito pouco lem sido publicado especificamente para a PRLP.

Passa-se a comentar alguns trabalhos de relevância que tentam suprir a lacuna acima apontada.

Monma \& Segal ${ }^{01}$ descrevem com detalhes um algoritmo simplex para encontrar fluxo de custo mínimo em redes capacitadas que se aplica, também, para o caso de função objetivo linear por partes. $\mathrm{O}$ algoritmo é de grande valia para redes de grande porte pelo seu excelente desempenho computacional, bem como possui caracteristicas importantes que evitam a ocorrência dos indesejáveis fenômenos de ciclagem ("cycling") e empacamento ("stalling"), decorrentes de situações de degenerescência: adicionalmente apresenta-se a estrutura de dados adotada na implementação de tal algoriluno.

Barras et al. apresentam uma formulação e as definições básicas para a PRLP, bem como um algoritmo tipo simplex para redes lineares por partes. Já o trabalho de Sun \& Tsai ${ }^{82}$ propõe um algoritmo simplex especializado para a PRLP, que é especialmente adequado para problemas com um número grande de termos lineares nas parcelas que compõem a função objetivo. O algoritmo é do tipo Big-M, com 
um mecanismo auto-adaptativo para escolha do número $M$. o que é realizado através do uso de uma fórmula empírica que depende fundamentalmente das inclinações minimas e máximas associadas às funções de custo lineares por partes em cada arco da rede.

Estes últimos autores estabelecem 0 seu método, analisam sua convergência, descrevem a estrutura de dados usada, e relatam una comparação compulacional do seu algoritmo com o bem conhecido algoritmo NETFLO, descrito em Kemington \& Helgason ${ }^{42}$. A aplicação do NETFLO se dá após a reformulação dos modelos de PRLP como modelos em redes lineares equivalentes. Estes modelos dos testes foram gerados pelo programa gerador de redes NETGEN de Klingman et al. ${ }^{44}$, que tem características interessantes, e tem sido adotado ell muitas pesquisas.

Na sua tese de doutoramento, Marins ${ }^{57}$. apresenta a formulação de programas em redes lineares por partes, a nolação adotada, e a fundamentação teórica do algoritmo implementado, que é uma especialização para a PRLP do método simplex para redes lineares proposto por Fourer $^{27}$.

Este algorimo possui caracteristicas especiais que permitem evitar a ciclagem e o empacamento, e tirar vantagem da estrutura linear por partes do problema para efetuar, numa dada iteração, uma exploração continuada de variável básica (não-básica) que permite a realização da maior umudança possivel no valor do fluxo do arco básico (não-básico) a ser substituido (considerado para entrar na base), caso se constate no teste da razão que este arco básico (não-básico) pode permanecer básico (não-básico) na próxima aplicação do método, sem haver mudança da àrvore geradora disponivel no momento. Ainda são apresentados resultados computacionais de testes do desempenho do algoritmo, quando combinado com várias regras de entrada de variável na base, incorporando ou não a abordagem pela técnica de mudança de escala, ou escalonamento dos dados ("scaling"), proposta por Edmonds \& Karp"1.

As descrições de três algoritmos especializados para modelos de PRLP. com suas caracteristicas e propriedades fundamentais, constam do artigo Marins \& Perin ${ }^{47}$ submetido à revista Pesquisa Operacional. Em outro trabalho, Marins el al.48 utilizam um experimento compulacional, com rigorosa base estatística, para comparar quatro algoritmos especializados para a PRLP, com respeito aos fatores a seguir, quando considerados individualmente ou em combinações: classe do problema, dimensão da rede, proporção de arcos capacitados na rede. e número de limitantes de intervalos de fluxo ("breakpoints") nos arcos da rede. Procura-se neste último traballo a resposta para as seguintes questões:

(1) Há um algoritmo que é sempre melhor que os demais testados. em qualquer situação? Ou altemativamente, qual algoritmo tem o melhor desempenho 


\section{PRODUÇÃO}

em cada categoria de modelos de PRLP considerados?;

(2) Quais sño os efeitos dos fatores considerados no desempenho de cada algoritmo?;

(3) Quais são os efeitos de mudanças conjuntas nos fatores estudados no desempenho de cada algoritmo?

Como já comentado, existem inúmeras aplicações da PRLP, passa-se a seguir a descrever brevemente algumas delas, que justificam a relevância da área.

Técnicas de Programação Matemática têm sido usadas com sucesso em variadas situações no campo de Sistemas de Potência, sendo que, particularmente, com frequiência o uso de modelos de PRLP no auxílio à solução de tais casos tem sido referenciado na literatura. Nesta linha, Sasson \& Merrill $^{76}$ elencam importantes problemas de planejamento e operação de sistemas de potência que podem ser tratados por meio de modelos de PRLP; já Barras et al. ${ }^{5}$ apresentam um algoritmo simplex para redes lineares por partes que se aplica ao problema do cálculo de fluxo de carga em corrente alternada e corrente contillua, sendo este problema de particular interesse, pois se constitui em parte importante de problemas mais gerais de segurança, e na análise de contingência, em sistemas de potência.

Uma outra área fértil na utilização de modelos de PRLP é no planejamento da operação de sistemas hidroelétricos de potência, nesta linha pode-se referenciar os artigos de Dembo et al. ${ }^{19}$, Rosenthal ${ }^{73}$, e Soares Filho et al. ${ }^{79}$, que descrevem para problemas reais resolvidos na Espanha. Estados Unidos da América, e Brasil, respeclivamente, a etapa de modelagem $\mathrm{e}$ a metodologia de solução adotada.

Os problemas surgidos na administ ração da Hidroeléırica Espanhola, uma das duas maiores daquele pais, sâo descritos em Dembo et a!. ${ }^{14}$. Na solução destes problemas os autores utilizaram modelos (para problemas com horizonte de planejamento de curlo prazo) que possibilitam o uso de técnicas de PRLP.

A referência Rosenthal ${ }^{73}$ descreve alguns problemas típicos na administração de sistemas de reservatórios, onde se busca a maximização dos benefícios proporcionados pelo sistema com respeito a várias características, tais como a existência de (a) um ou vários reservatórios; (b) periodo único ou multiperiodos; (c) fluxo determinístico ou estocástico; e (d) função objelivo linear, não-linear separável e não separável. O artigo também relaciona vários métodos para a solução destes problemas, onde se inclui a aproximação por funções lineares por partes para o caso de fuução objetivo separável

Soares Filho et al. ${ }^{79}$ descrevem uma aplicação ao planejamento multiobjetivo de médio prazo da operação de um sistema de mullireservatórios. O sistema analisado é o da bacia do rio Piracicaba, no estado de São Paulo. Os objetivos a serem maximizados estão associados a dois usos conflitantes da água da região: suprimento 


\section{PRODUÇÃO}

e qualidade. Outros aspectos como produção de energia elétrica e controle de cheias são considerados como limitantes sobre o nivel de água armazenada e a descarga necessária de água nos reservatórios. O método aplicado representa este problema, com respeito a três cenários hidrológicos - sêco, úmido e em condições médias, como um problema en rede linear por partes, considerando a estrutura da rede liidráulica associada ao sistema de multireservatórios estendida no tempo.

Também em sistemas de telecomunicações tem naturalmente surgido aplicações da PRLP. Três casos típicos sĩo descritos em Monma \& Segal ${ }^{\text {bl }}$. um problema de transferência de facilidades entre áreas, um problema de programação da coleta de registros de chamadas numa rede privada de comunicação, e um problema de planejamento da expansão de capacidade de sistemas de telecomunicações. Estes autores também propõem um algoritmo eficiente para solucionar modelos em redes lineares por partes. Também França et al.$^{29}$ e Nakagawa ${ }^{63}$ tratam de casos similares, relativos à expansão de redes telefônicas, e alocação de centros de fios, respectivamente.

Outra siluação, onde o uso de modelos de PRLP é de muila valia, se refere ao problema clássico da análise de redes de tubulações. que busca encontrar o conjunto de fluxos e pressões numa rede de distribuição de água, conhecidos os niveis de suprimento e de demanda de agua; a solução deste problema fornece informações básicas para todo o projeto de engenharia do sistema de distribuição de água. Collins et al. ${ }^{12}$ relatam o estudo de um caso deste tipo envolvendo a análise da rede de tubulações da cidade de Dallas nos Estados Unidos da América.

$\mathrm{Na}$ área de administração de projetos pode-se citar dois artigos interessantes, Ahuja et al. ${ }^{2}$ e Levner \& Nemirovsky ${ }^{40}$, que abordam, respectivamente. o problema de negociação entre duração do projeto $\mathrm{e}$ seu custo ("time-cost tradeoff problem") numa rede CPM (ver Ravindran et al ${ }^{\circ 8}$ para uma descrição do caso), e o problema da programação de projetos com objetivo e restrições orientadas por uma política tipo "just-in-time". proposto por Anthonisse et al. ${ }^{4}$.

Quanto a sistemas de transportes, a dificuldade está em selecionar exemplos de aplicação da PRLP, devido a enormc quantidade e variedade destas que têmı sido reportadas na literatura cientifica. Passi-se a comentar, sucintamente, alguns tipos de problemas de transporte que se enquadram claramente numa abordagem pela PRLP.

O primeiro deles, tratado em Klein ${ }^{43}$. é o do modelo de transporte que admite o custo de planejamento da produção como sendo convexo, respeitando restrições de estoque e com possibilidade de haver ordens pendentes de pedidos.

Outros dois casos estão associados a existência de incerteza nas demandas, tanto 
num problema de transporte clássico, como em Cooper \& LeBlanc ${ }^{14}$, e em Ferguson \& Dantzig ${ }^{22}$, como num problema que admita nós de transbordo ("transshipment nodes") tal como relatado em Sun et al. ${ }^{83}$.

Finalmenle. com respeito a outro importante caso dentro do estudo de sistemas de transportes, a publicação de LeBlanc ${ }^{45}$ apresenta a utilidade da PRLP para a solução de modelos de equilibrio em redes de tráfego, adimitindo demandas fixas ou variáveis.

\section{Comentários Finais}

O objetivo principal desta pesquisa foi o de propiciar aos interessados ell siluações em que modelos de PLP e de PRLP possam ser íteis, informações acerca de quais algoritmos estão disponiveis para serem aplicados na solução de seus modelos, onde encontrá-los, sua descrição, caraclerísticas, e também qual o desempenho relativo de cada um destes métodos frente a diferentes categorias de problemas e de perfis de dados.

A constatação da importância da PLP e da PRLP se deu duplamente, lanto por meio do interesse demonstrado por ont ros pesquisadores, e também profissionais de várias áreas, quando da divulgação dos resultados parciais da pesquisa em eventos científicos, ver Marins \& Perin ${ }^{50.53 .54 .55 .56} \mathrm{e}$ Marins et al ${ }^{49,51,52}$, como por intermédio dos resultados obtidos tia pesquisa bibliográfica realizada pelos autores na literatura disponível.

Esse Icvantamento bibliográfico. que foi a base deste texto, incluiu a consulta às mais importantes revistas de divulgaçĩo cientifica internacionais, várias teses de autores nacionais e estrangeiros. relatórios técnicos e também inúmeros contatos (pessoais e por correspondências) realizados com pesquisadores de destaque na área.

Assim, pelo que já foi anteriormente exposto, acredita-se que esteja plenamente justificada a validade deste trabalho, a motivação para se continuar o desenvolvimento de pesquisa nas áreas da PLP e da PRLP, bem como a importância intrínseca deste esforço para avanços significativos destas áreas.

Agradecimentos: $\dot{A}$ FAPESP Fundação de Amparo à Pesquisa do Estado de São Paulo (processos nimeros 93/3441-2, 94/0459-0) c ao CNPq - Conselho Nacional de Pesquisa e Desenvolvimento (processo número 350330/94-8). 


\section{Referências bibliográficas}

[1] Alllfeld, D. P., R. S. Dembo, J. M. Mulvey, e S. A. Zenios. 1987. Nonlinear Programming on Generalized Networks. Association for Computing Machinery Transactions on Mathematical Sofiware 13. No. 4. 35()-367.

[2] Alujia. R. K.. J. L. Batra, e S. K. Gupta. 1984. A Parametric Algorithm for the Convex Cost Network Flow and Related Problems. European Journat of Operational Research 16, No. 2, 222-235.

[3] Alı!ja, R. K., T. L. Magnanti, e J. B. Orlin. 1993. Network Flows: Theory, Algorithms and Applications. Englewood Cliffs. NJ. Prentice-Hall.

[4] Anthonisse, J. M., K. M. Van Hee, e J. K. Lenstra. 1988. ResourceConstrained Project Scheduling: An International Exercise in DSS. Decision Support Sivstems 3, 249-257.

[5] Barras, J., S. Alec, C. Pasche, P. A. Chamorel. A. J. Germond, e D. De Werra. 1987. Network Simplex Method Applied to AC Load-Flow Calculation. Institute of Electrical and Electronics Engineers Transactions on Power Systems Vol PWRS-2. No. 1, 197-2013.

[6] Barrodale, I.. e F. D. K. Roberts. 1973. An Inproved Algorithm for Discrete $\mathrm{L}_{1}$ Linear Approximation. SLAM Journal on Numerical Analysis 10, 839-848.
[7] Bartels, R. H. 1980. A Penalty Linear Programming Method Using ReducedGradient Basis-Exchange Techniques. Linear Algebra and Its Applications 29. 17-32.

|8| Beale. E. M. L. 1959. An Algorithm for Solving the Transportation Problem when Shipping Cost over each Route is Convex. Naval Research Logistics Quarterly6. 43-56.

[9] Charnes, A., e C. E. Lennke. 1954 Minimization of Non-Linear Separable Convex Functionals. Naval Research Logistics Quarterly 1, 301-312.

[10] Charnes, A., e W. W. Cooper. 1957. Nonlinear Power of Adjacent Extreme Point Methods in Linear Programming. Econometrica 25. 132-153.

[11] Charret, D. E. 1970. Plastic Analysis and Optimum Design of Slabs and Frames. Special Report S2/1970, Civil Engineering, Monash University. Clayton - Vicloria, Australia.

[12] Collins, M.. L. Cooper R. Helgason. J. Kennington. e L. LeBlanc. 1978. Solving the Pipe Network Analysis Problem Using Optimization Techniques. Management Science 24. No. 7. 747-760.

113] Conn, A. R. 1976. Linear Programming via a Nondifferentiable 
Penalty Function. SIAM Journal on Numerical Analysis 13, No. 1, 145-154.

[14] Cooper, L., e L. J. LeBlanc. 1977. Stochastic Transportation Problems and Other Networks Related Convex Problems. Naval Research Logistics Quarterly 24, No. 2, 327-337.

[15] Dantzig, G. 1956. Recent Advances in Linear Programming. Management Science 2, No. 2, 131-144.

[16] Dantzig, G. B. 1955. Linear Programming under Uncertainty. Management Science 1, No. 3, 197-206.

[17] Dantzig, G., S. Johnson, e W. White. 1958. A Linear Programming Approach to the Chemical Equilibrium Problem. Management Science 5, No. 1, 38-43.

[18] Davies, M.. 1967. Linear Approximation Using the Criterion of Least Total Deviations. Journal of the Royal Statistical Society B 29, 101-109.

[19] Dembo, R. S., A. Chiarri, J. G. Martin, e L. Paradinas. 1990. Managing Hidroeléctrica Española's Hydroelectric Power System. Interfaces 20, No. 1, 115135.

[20] De Wolf, D., O. J. De Bisthoven, e Y. Smeers. 1991. The Simplex Algorithm Extended to Piecewise Linearly Constrained Problems I: the Method and an Implementation. Discussion Paper No. 9119. Center for Operations Research \& Econometrics, Universite Catholique de Louvain, Belgium.
[21] Edmonds, J., e R. M. Karp. 1972. Theoretical Improvements in Algorithmic Efficiency for Network Flow Problems. Journal of the Association for Computing Machinery 19, No. 2, 248-264.

[22] Ferguson, A. R., e G. B. Dantzig. 1957. The Allocation of Aircrafts to Routes: An Example of Linear Programming under Uncertain Demand. Management Science 3, No. 1, 45-73.

[23] Fernandes, J. F. R. 1979. Programação Linear por Partes: Um Problema de Grande Porte Resolvido por Técnicas de Decomposição. Tese de Doutoramento, Faculdade de Engenharia Elétrica, Universidade Estadual de Campinas, Campinas - SP, Brasil.

[24] Ferreira, E. P. 1979. Programação Linear por Partes: Um Mélodo Duad-Primal. Dissertação de Mestrado, Faculdade de Engenharia Elétrica, Universidade Estadual de Campinas, Campinas - SP, Brasil.

[25] Fourer, R. 1992. A Simplex Algorithm for Piecewise-Linear Programming III: Computational Analysis and Applications. Mathematical Programming 53, No. 2, 213-235.

[26] Fourer, R. 1988. A Simplex Algorithm for Piecewise-Linear Programming Il: Finiteness, Feasibility and Degeneracy. Mathematical Programming 41, No. 3, 281-315.

[27] Fourer, R. 1985. A Simplex Algorithm for Piecewise-Linear 
Programming I: Derivation and Proof. Mathematical Programming 33, No. 2, 204233.

[28] Fourer, R., e R. E. Marsten. 1992. Solving Piecewise-Linear Programs: Experiments with a Simplex Approach. ORSA Journal on Computing 4, No. 1, 1531.

[29] França, P. M., J. F. R. Fernandes, e H. M. F. Tavares. 1987. Telephonic Network Expansion. Controle \& Automação I, No. 3.

[30] Garcia, A. S. 1978. Um Método Dual-Simplex para Programação Linear por Partes. Dissertação de Mestrado, Instituto de Matemática, Estatística e Ciências da Computação, Universidade Estadual de Campinas, Campinas - SP, Brasil.

[31] Geoffrion, A. M. 1977. Objective Function Approximations in Mathematical Programming. Mathematical Programming 13, No. 1, 23-37.

|32| Glover, F., D. Klingman, e D. T. Phillips. 1992. Networks Models in Optimization and Their Applications in Practice. NY, John Wiley \& Sons.

[33] Glover, F., D. Klingman, e N. Phillips. 1990. Netform Modeling and Applications. Interfaces 20, No. 4, 7-27.

|34| Golstein, E. G. 1960. A Certain Class of Nonlinear Extrenum Problems. Soviet Mathematics 4, 863-866.
[35] Golstein, E. G., e D. Youdine. 1973. Problèmes Particuliers de la Programmation Lineaire. Moscow. Editions Mir.

|36| Güder. F.. c J. G. Morris. 1994. Optimal Objective Function Approximation for Separable Convex Quadratic Programming. Mathematical Programming 67. No. 1. 133-1+2.

137] Gunn, E. A. .1981. An Unconstrained Dual for Linear Programming and its Solution by Piecervise Linear Optimization. Utilitas Mathematica 20,5-19.

[38] Ho, J. K. 1985. Relationship Among Linear Formulation of Separable Convex Piecewise-Linear Programs. In: Mathematical Programming Sudy 24. 126-140, edited by R. W. Cottle, Amsterdam - The Netherlands, North-Holland Publishing Company.

[39] Hochbaum, D. S., e J. G. Shanthikumar. 1990. Convex Separable Optimization Is Nol Much Harder than Linear Optimization. Journal of the Association for Computing Machinerv 37. No. $4,8+3-862$.

[40] Kamesam, P. V.. e R. R. Meyer. 1984. Multipoint Methods lor Separable Nonlinear Networks. In: Mathematical Programming Studly 22, 185-205.

141] Kao, C. Y., e R. R. Meyer. 1981. Secant Approximation Methods for Convex Optimization. In: Mathematical Programming Situdy 14, 143-162, edited by 


\section{PRODUÇÃO}

H. Hönig, B. Korte, e K. Ritter, Amsterdam

- The Netherlands, North-Holland Publishing Company.

[42] Kennington, J. L., e R. V. Helgason. 1980. Algorithms for Network Programming. NY., John Wiley \& Sons.

|43| Klein, M.. 1983. A Transportation Model for Production Planning with Convex Costs. IIE Transactions 15, No. 3, 272-274.

[44] Klingman, D., A. Napier, e J. Stutz. 1974. NETGEN: A Program for Generating Large Scale Capacitated Assignment, Transportation, and Minimum Cost Flow Network Problems. Management Science 20, No. 5,814-821.

[45] Leblanc, L. J. 1976. The Use of Large Scale Mathematical Programming Models in Transportation Systems. Transportation Research 10, No. 6, 419-421.

[46] Levner. E. V., e A. S. Nemirosvsky. 1994. A Network Flow Algorithm for Justin-Time Project Scheduling. European Journal of Operational Research 79, 167175.

[47] Marins, F. A. S., e C. Perin Filho 1996. Algorilmos para a Programação em Redes Lineares por Partes. Submetido para publicação na revista Pesquisa Operacional da SOBRAPO.

[48] Marins, F. A. S., K. Darby-Downman, E. F. Seme, A.F. Machado, e C. Perin Fillıo. 1996. Algorillums for Network Piecewise-
Linear Programs: A Statistically Designed Study. Aceito para publicação no European Journal of Operational Researclı.

[49] Marins. F. A. S., K. Darby-Downian, E. F. Selue, A.F. Machado, e C. Perin Filho. 1995. Algorithuns for Network PiecewiseLinear Programs: A Comparative Study. Anais do XXVII Simpósio Brasileiro de Pesquisa Operacional, Vitória - ES, Brasil.

[50] Marins, F. A. S., e C. Perin Filho. 1994. Algorithms for Nelwork PiecewiseLinear Programs. Anais de Resumos do EURO XII/OR 36, Glasgow - Escócia.

[51] Marins, F. A. S.. C. Perin Filho, e A. F. Machado. 1993. An O(n.m' $\log$ n. min $(\log \mathrm{nC}, \mathrm{m} ' \log n))$ Algorithm for Network Piecewise-Linear Progranıs. Aulais do XXV Simpósio Brasileiro de Pesquisa Operacional, Campinas - SP, Brasil.

[52] Marins, F. A. S., C. Perin Fillo, e A. F. Machado. 1992. A Strongly Polynomial Algorithm for Network Piecervise-Linear Prograns. Apresentado no XXIV Simpósio Brasileiro de Pesquisa Operacional, Salvador - BA. Brasil.

153| Marins, F. A. S. e C. Perin Filho. 1991. Computationtal Experience with a Dual Algorithm for Network Piecewise-Linear Programs. Anais do XIII World Congress on Computational and Applied Mathematics, 197-198, Dublin, Irlanda.

[54] Marins, F. A. S., e C. Perin 1990. A Dual Algorithm for Network Piecewise- 
Linear Programs. Apresentado no V Congresso Latino-Ibero-Americano de Pesquisa Operacional e Engenharia de Sistemas, Buenos Aires, Argentina.

[55] Marins, F. A. S., e C. Perin Filho 1989. An Out-of-Kilter Algorithm for Network Piecewise-Linear Programs. Anais do XII Congresso Nacional de Matemática Aplicada e Computacional, 126-130, São José do Rio Preto - SP, Brasil.

[56] Marins, F. A. S., e C. Perin Filho 1988. Strong Feasibility in Network Piecewise-Linear Programs. Anais do IV Congresso Latino-Ibero-Americano de Pesquisa Operacional e Engenharia de Sistemas, 364-383, Rio de Janeiro - RJ, Brasil.

[57] Marins, F. A. S. 1987. Estudos de Programas Lineares por Partes. Tese de Doutoramento, Faculdade de Engenharia Elétrica, Universidade Estadual de Campinas, Campinas - SP, Brasil.

[58] Markowitz, H. M., e A. F. Perold. 1981. Sparsity and Piecewise Linearity in Large Portfolio Optimization Problems. In: Sparse Matrices and Their Uses, 89-108, edited by I. S. Duff, Academic Press, NY.

[59] Meyer, R. R. 1982. Recursive Piecewvise-Linear Approximation Methods for Nonlinear Networks. In: Evaluating Mathematical Programming Techniques, Lecture Notes in Economics and Mathematical Systems Vol. 199, 315-322, edited by J. M. Mulvey, Springer-Verlag, NY.
[60] Meyer, R. R. 1979. Two-Segment Separable Programming. 1979. Management Science 25, No. 4, 385-395.

[61] Momma, C. L., e M. Segal. 1982. A Primal Algorithm for Finding MinumumCost Flows in Capacitated Networks with Applications. The Bell System Technical Journal 61, №. 6, 949-968.

[62] Mulvey, J. M. 1989. Advances in Nonlinear Network Models and Algorithmis. 1n: Algorithms and Model Formulations in Mathematical Programming - NATO ASI Series Vol F5I, edited by Stein W. Wallace, SpringerVerlag, Berlin.

[63] Nakagawa, J. M. 1984. Planejamento de Sistemas Telefônicos: Alocação de Centros de Linhas. Dissertação de Mestrado, Faculdade de Engenharia Elétrica, Universidade Estadual de Campinas, Campinas - SP, Brasil.

[64] Orchard-Hays, W. 1968. Actranced Linear-Programming Computing Techniques. McGraw-Hill, New York, NY.

[65] Orden, A., e V. Nalbandian. 1968 A Bidireclional Simplex Algorilhm. Journal of the Association for Computing Machinery 15, No. 2, 221-235.

[66] Perold, A. F. 1984. Large-Scale Portfolio Optimization. Management Science 30, No. 10, 1143-1160.

[67] Premoli, A. 1986. Piecewise-Linear Programming: The Compace (CPLP) 
Algorithm. Mathematical Programming 36. No. 2, 210-227.

[68] Ravindran, A., D. T. Phillips, e J. J. Solberg. 1987. Operations Research Principles and Practice. New York, NY, John Wiley \& Sons.

[69] Ribeiro, R. V. 1980. Esludos ell Programação Linear. Tese de Doutoramento, Faculdade de Engenharia Elétrica, Universidade Estadual de Campinas, Campinas - SP, Brasil.

[70] Robers, P. D., e A. Ben-Ismel. 1969. An Interval Programming Algorithm for Discrete Linear $L_{1}$ Approximation Problems. Journal of Approximation Theory 2, 323336.

171] Rockafellar, R. T. 1984. Network Flows and Monotropic Optimization. Wiley-Interscience, New York.

[72] Rockafellar, R. T. 1981. Monotropic Programming: Descent Algorithms and Duality. In: Nonlinear Programming 4, 327-366, edited by O. L. Mangasarian, R. R. Meyer. e S. M. Robinson. NY, Academic Press.

[73] Rosenthal, R. E. 1981. A Nonlinear Network Flow Algorithm for Maximization of Benefits in a Hydroelectric Power System. Operations Research 29, No. 4, 763-786.

[74] Rosvany. G. I. N. 1971. Concave Programming and Piecewise-Linear
Programming. International Journal for Numerical Methods in Engineering 3, No. $1,131-144$.

175] Rosvany. G. I. N. 1970. Concave Programming in Structural Optimization. International Journal of Mechanical Sciences 12, No. 2, 131-142.

[76] Sasson, A. M., eH. D. Merrill. 1974. Some Applications of Optimization Techniques to Power Systems Problems. Proceedings of the Institule of Electrical and Electronics Engineers 62, No. 7, 959972.

[77] Snyder, R. D. 1984. Linear Programming with Special Ordered Sets. Journal of the Operational Research Societv 35, No. 1, 69-74.

178| Snyder, R. D. 1981 Programming with Piecewise-Linear Objective Functions. Working Paper 9/81. Departuent of Econometrics and Operations Research. Monash University, Clayton, Victoria, Australia.

|79| Soares Filho, S., B. P. F. Braga Jr., e J. G. L. Caneja. 1989. Mid Term Multiobjective Operation Planning of a Multireservoir System: The Piracicaba River Basin Case. Apresentado no International Symposium on Water Resource Systems Application, Cantada.

[80] Souza, C. R. 1977. Uma Aplicação de Programação Linear por Partes a Sistemas de Potência. Dissertação de Mestrado. Faculdade de Engenharia Elétrica, Universidade Estadual de Campinas, Campinas - SP, Brazil. 
[81] Spyropoulos, K., E. Kiountouzis, e A. Young. 1973. Discrete Approximation in the $L$, Norm. The Computer Journal 16, 180-186.

[82] Sun, J., e K. Tsai. 1989. An Inplementation of the Network Simplex Method for Piecewise-Linear Programs. Technical Report 89-07, Department of Industrial Engineering and Management Sciences, Northwestern University, Evanston, IL, USA.

[83] Sun, J., L. Qi, e K.-H. Tsai. 1989. Solving Stochastic Transshipment Problems as Network Piecewise Linear Programs. Technical Report, Department of Industrial Engineering and Management Sciences. Northwestern University, Evanston, IL, USA.

[84] Thakur, L. S. 1986. Successive Approximation in Separable Progranuming: An Improved Procedure for Convex Separable Programs. Naval Research Logistics Quarterly33, No. 2, 325-358.

[85] Thakur, L. S. 1978. Error Analysis for Convex Separable Programs: the Piecewise-Linear Approximation and the Bounds on the Optimal Objective Value. SIAM Journal on Applied Mathematics 34, No. 4, 704-714.

[86] Veldhorst, M.. 1990. A Bibliography on Network Flow Problems. Algorithms Review 1, No.2, 97- 117.

[87] Williams, A. C. 1962. A Treatment of Transportation Problems by
Decomposition. SIAM Journal 10. No. 1. 35-48.

[88] Wolfe, P. 1965. The Composite Simplex Algorithm. SLAM Review 7, 42-54.

Departamento de Produção. Faculdade de Engenharia do Campus de Guaratinguetá - UNESP. CEP 12500-(00). Guaratinguetá, SP, Brasil.

Departamento de Matemática Aplicada. Instituto de Matemálica c Estatística, UNICAMP, CEP 13081-970, Campinas, SP. Brasil. 